\title{
Irritable bowel syndrome among paramedical students, King Abdulaziz University, Jeddah, Saudi Arabia
}

\author{
Nahla Khamis Ibrahim ${ }^{1,2 *}$, Shatha Al-Jamhoor ${ }^{3}$, Nashwa Ashour ${ }^{3}$, Amjaad Alsulami $^{3}$, Dania Bukhari ${ }^{3}$, Ahlam Al-bloshi ${ }^{3}$ and Traji Fathi $^{3}$ \\ ${ }^{1}$ Department of Family and Community Medicine Department, King Abdulaziz University, Jeddah, Saudi Arabia \\ ${ }^{2}$ Department of Epidemiology, High Institute of Public Health, Alexandria University, Alexandria, Egypt \\ ${ }^{3}$ Six Year Medical Student, King Abdulaziz University, Jeddah, Saudi Arabia
}

\begin{abstract}
Aim: The study was done to determine the prevalence and associated factors of IBS among paramedical students at King Abdulaziz University, Jeddah, Saudi Arabia.

Methods: A cross-sectional study was done during 2016/2017 at the Faculty of Health Sciences, King Abdulaziz University, Jeddah. A multistage stratified random sample method was used to select 525 paramedical students. A standardized self-administered data collection sheet was used and contained the validated scales of Rome III Criteria, Hospital Anxiety and Depression Scale (HADS) and Pittsburgh Sleep Quality Index (PSQI).

Results: One-third of the paramedical students met the Rome-III criteria of IBS. Nursing and dentistry students had higher IBS prevalence compared to others. IBS-Mixed and IBS-Constipation were the commonest sub-types. IBS was associated with female gender, educational specialty, family history of IBS, family income, chronic medical conditions, food hypersensitivity, traveler's diarrhea, poor sleep quality, stress, anxiety, and depression. Regression analysis revealed that female gender (aOR: 1.83; 95\% CI: 1.12-3.28), stress, depression, IBS family history and poor sleeping were IBS predictors after controlling of confounding factors.
\end{abstract}

Conclusion: The prevalence of IBS was high among paramedical students, and it was predicted by gender, stress, depression, poor sleeping, and family history. Screening for IBS and psychological problems, stress management courses and IBS educational programs are recommended for paramedical students.

\section{Introduction}

Irritable bowel syndrome (IBS) is a global, wide-spreading, costly and disabling chronic Functional Gastrointestinal Disorder (FGID). It is the most frequently diagnosed type of the FGIDs, and the commonest reason for visiting gastroenterologist $[1,2]$. The condition is manifested by alteration of bowel habits (diarrhoea and constipation), abdominal pain and bloating, in absence of chemical or pathological disorders and other red flags. IBS is a sensory-motor illness, with a multi-factorial aetiology. Motility disorders, alteration of the gut mucosal immune activity, visceral hypersensitivity, central processing dysfunctions, psychological factors, and infections play roles in IBS aetiology $[1,3,4]$.

IBS is associated with somatic co-morbidities (as over-active bladder, migraine and painful syndromes). Similarly, it can be associated with visceral sensitivity and psychological conditions (anxiety and depression). Furthermore, many physiological, psychological and stresses factors can play a role in severity of IBS symptoms. Stresses usually worsens gastrointestinal symptoms [5]. IBS condition can be only diagnosed clinically; as no biomarkers or confirmative investigations available for it $[1,2]$.

It was estimated in 2014 that about $11 \%$ of the global population are affected by IBS [6]. A study done between university students from Lebanon revealed that more than half of the diagnosed students with IBS were enrolled in the Faculty of Medical Sciences (Medicine, Pharmacy, and Dentistry) [7]. Ibrahim et al. [8] reported that IBS affected 31.8\% of medical students and interns from the main governmental medical college in Jeddah, Saudi Arabia. Students enrolled in faculties working in the medical fields are usually exposed to various stressors such as academic stresses and an uncertain future. They also represent a large and important sector of the future health team $[1,8]$.

However, insufficient number of studies had been conducted to illustrate the problem of IBS between paramedical students in Jeddah. So, such study is needed. The current study was done to determine the prevalence and associated factors of IBS between paramedical students at King Abdulaziz University (KAU), Jeddah, Saudi Arabia.

\section{Methods}

The study was done using a cross-sectional approach during 2016/2017. It was done among paramedical students enrolled in faculties of Nursing, Dentistry and Applied Medical Sciences (Nutrition, Physiotherapy \& Laboratory specialities). A multistage stratified random sample method was used during 2015/2016. Stratification considered the gender, educational level, faculty and specialty. All those who finished freshman year and accepted to participate enrolled in the study.

Correspondence to: Nahla Khamis Ibrahim, Department of Family and Community Medicine, King Abdulaziz University, Jeddah, Saudi Arabia, Tel: 00966501632237; E-mail: nahlakhamis@yahoo.com

Key words: irritable bowel syndrome; stress; depression; sleep quality; paramedical students s, Rome III criteria info.medicinearticle@gmail.com, prevalence, predictors

Received: February 13, 2018; Accepted: February 20, 2018; Published: February 27, 2018 


\section{The sample was calculated according to the equation [9]}

$$
\text { "n= } \mathrm{Z}^{2} \times \mathrm{P} \times \mathrm{q} "
$$

$\mathrm{d} 2$

Prevalence (p) was set as $31.8 \%$; based on to the latest study from Jeddah. ${ }^{8}$ The minimal sample that was needed for achieving a precision of $\pm 4 \%$ at $95 \%$ confidence interval (CI) amounted to 521 participants, which was rounded to 525 .

A constructed, validated, data collection sheet was utilized. Face and content validity were assessed by 2 experts. Internal-consistency reliability was found to be $81 \%$.

Personal, socio-demographic information, lifestyles, stresses during the six-months preceded the study, and family history of IBS were determined. Students were also asked about prior diagnosis of IBS and other chronic medical conditions including traveler's diarrhoea. Weight and height of the students were assessed. Rome III criteria was utilized to identify IBS cases, and its sub-types [10]. Students also completed the standardized version of the Hospital Anxiety and Depression Scale (HADS) [11] and the Food Frequency Questionnaire (FFQ). Pittsburgh Sleep Quality Index (PSQI) [12] was completed. All the used scales have high validity and internal consistency reliability [10-12].

\section{Statistical analysis}

Data was analysed by SPSS Version 20. Different scores were calculated as followings:

Rome III criteria was used to classify the participants into those who met IBS criteria or not. It then divided the detected IBS cases according to their sub-types into IBS-Constipation (IBS-C), IBS-Diarrhoea (IBS-D), IBS-Mixed (IBS-M) and IBS-Unsub-typed (IBS-U) [10].

Body Mass Index (BMI): It was calculated (Weigh in kilogram/ Height ${ }^{2}$ in meter $^{2}$ ), and then it was categorized into: normal weight or excessive weight (over-weight and obese) [8].

Hospital Anxiety and Depression (HADS): A self- rating scale of 14 items; 7 items for anxiety HADS-Anxiety (HADS-A) and 7 for HADS- depression (HADS- depression). It was then categorized into normal (from 0-7), borderline case (from 8-10) and abnormal (from 11-21). Then it was divided into either normal or abnormal [11].

PSQI: It consists of 19 items; which is grouped into seven components. Participants who obtained a score higher than 5 were then considered poor sleepers [12].

Descriptive and inferential statistics were done. A multiple logistic regression model was constructed to determine IBS predictors after controlling confounding. Adjusted Odds Ratio (aOR), and 95\% confidence Intervals (CI) were calculated. All $p$-values $<0.05$ were considered statistically significant.

Ethical statement: The study followed the ethical standards of Helsinki Declaration. Approval of conduction of the research was taken from the Ethics and Research Committee of KAU. A written informed consent was obtained from each participant.

\section{Results}

A total of 525 paramedical students enrolled in the study. Their age ranged from 18-28 years with a mean of $21.38 \pm 2.09$. Analysis of PSQI revealed that the prevalence of poor sleep quality among all paramedical students was $68.6 \%$.
Table 1 illustrates that $33.3 \%$ of the paramedical students met RomeIII criteria for IBS diagnosis. IBS-Mixed (IBS-M) was the commonest (58.9\%) prevalent sub-type. On the other hand, IBS-Constipation (IBS-C), IBS- Unsubtyped (IBS-U), and IBS-Diarrhoea (IBS-D) accounted for $15.4 \%, 14.3 \%$ and $11.4 \%$ of the cases, respectively. Out of 175 cases diagnosed as IBS in the current analysis, only 42 students $(24.0 \%)$ were previously diagnosed as having IBS by physician (s).

Table 2 illustrates that the prevalence of IBS was much higher among females (38.1\%) compared to males (20.8\%). A highly statistical significant difference was present $\left(X^{2}=13.95, p<0.0001\right)$. Regarding educational specialty, nursing (41.7\%) and dentistry (37.8\%) students had the highest IBS prevalence compared to others. On the other hand, students from the nutritional specialty had the lowest prevalence. IBS was significantly associated with family history of the condition $(p<0.001)$. Older students and those lived in the university dorms had higher IBS prevalence than others $(p>0.05)$.

It is apparent from table 3 that IBS was significantly associated with presence of chronic medial diseases $(O R=2.19$; 95\% CI: 1.33-3.61). It is also associated with traveler's diarrhea. In addition, participants who had food hypersensitivity reported a significantly higher prevalence of IBS (45.1\%) compared to others (31.5\%). Students who faced stress, during the six months preceded the study, were about 2.5 times more prone to IBS compared to others ( $O R=2.19$; 95\% CI: 1.33-3.61). Furthermore, IBS was higher among students diagnosed as having border-line and morbid anxiety (HAS-A) compared to normal students $(p<0.05)$. Similarly, IBS prevalence was significantly higher $(42.2 \%)$ among students with borderline and morbid depression (HAS-D) compared to others (27.4\%). Poor sleepers were about 2 times more liable to IBS than others (OR=1.94; 95\% CI: 1.33-2.83). Smokers, those with higher BMI, and those didn't practice physical exercise had higher prevalence of IBS compered to others, but without statistical significant differences $(p>0.05)$.

Analysis of FFQ showed that there are no associations between different dietary intake and IBS.

Controlling confounding factors in logistic regression analysis (Table 4) reveals that gender (female) was the first predictor of IBS (aOR: 2.11; 95\% CI: 1.30-3.41). Stress during the six months preceded the study (aOR:1.83; 95\% CI: 1.12-3.28), diagnosis of depression (aOR: 1.74; 95\% CI: 1.12-2.36). Presence of family history of IBS (aOR: 1.64; 95\% CI: 1.11-2.36), and poor sleep quality (aOR: 1.57; 95\% CI: $1.05-$ 2.35) were the following IBS predictors.

Table 1. Prevalence, subtypes of IBS among paramedical students at King Abdulaziz University.

\begin{tabular}{|l|c|c|}
\hline Irritable bowel syndrome & Number & Percent \\
\hline No Irritable Bowel syndrome & 350 & 66.6 \\
\hline Irritable Bowel syndrome & 175 & 33.3 \\
\hline Total & 525 & 100 \\
\hline Types of IBS (Total=175) & 103 & 58.9 \\
\hline IBS- Mixed (IBS-M) & 27 & 15.4 \\
\hline IBS-Constipation (IBS-C) & 25 & 14.3 \\
\hline IBS Unsubtyped (IBS-U) & 20 & 11.4 \\
\hline IBS-Diarrhea (IBS-D) & 42 & \\
\hline Previously diagnosed by a physician as IBS & & 24 \\
\hline Yes & 133 & 76 \\
\hline No & 175 & 100 \\
\hline Total IBS diagnosed cases & & \\
\hline
\end{tabular}


Table 2. Relationship between personal, socio-demographic factors, family history and irritable bowel syndrome among paramedical students at King Abdulaziz University. N.B.: RC: Referent category.

\begin{tabular}{|c|c|c|c|c|c|c|}
\hline \multirow{2}{*}{ Variable } & $\begin{array}{c}\text { IBS } \\
(\text { No. }=175)\end{array}$ & $\begin{array}{c}\text { No-IBS } \\
(\text { No. }=\mathbf{3 5 0})\end{array}$ & \multirow[t]{2}{*}{$\mathbf{X}^{2}$} & \multirow[t]{2}{*}{$\mathbf{P}$} & \multirow{2}{*}{ OR } & \multirow[t]{2}{*}{$95 \% \mathrm{CI}$} \\
\hline & No. $\%$ & No. $\%$ & & & & \\
\hline \multicolumn{7}{|l|}{ Age } \\
\hline$\leq 20$ & $53(29.3)$ & $128(70.7)$ & 0.062 & 0.15 & 0.75 & $0.51-1.11$ \\
\hline$>20$ & $122(35.5)$ & $222(64.5)$ & & & & \\
\hline \multicolumn{7}{|l|}{ Gender } \\
\hline Female & $145(38.1)$ & $236(61.9)$ & 13.95 & 0 & 2.33 & $1.49-3.67$ \\
\hline Male & $30(20.8)$ & $114(79.2)$ & & & & \\
\hline \multicolumn{7}{|l|}{ Educational specialty } \\
\hline Nursing & $30(41.7)$ & $42(58.3)$ & & & 3.57 & $1.57-8.15$ \\
\hline Dentistry & $37(37.8)$ & $61(62.2)$ & & & 3.03 & $1.37-6.69$ \\
\hline Laboratory & $31(36.0)$ & $55(64.0)$ & & & 2.82 & $1.25-6.33$ \\
\hline Physiotherapy & $34(33.0)$ & $69(67.0$ & 11.13 & 0.04 & 2.46 & $1.11-5.45$ \\
\hline Pharmacy & $33(31.1)$ & $73(68.9)$ & & & 2.26 & $1.02-4.99$ \\
\hline Nutrition & $10(16.7)$ & $50(83.3)$ & & & $1^{\mathrm{RC}}$ & \\
\hline \multicolumn{7}{|l|}{ Income } \\
\hline Enough \& exceeds & $83(29.1)$ & $202(70.9)$ & 4.55 & 0.03 & 0.67 & $0.47-0.96$ \\
\hline Enough only or debt & $91(52.3)$ & $149(42.5)$ & & & & \\
\hline \multicolumn{7}{|l|}{ Living place } \\
\hline With family & $158(33.0)$ & $321(67.0)$ & 0.514 & 0.77 & 0.7 & $0.26-1.88$ \\
\hline Private dorm & $10(34.5)$ & $19(65.5)$ & & & 0.75 & $0.22-2.58$ \\
\hline University dorm & $7(41.2)$ & $10(58.8)$ & & & $1^{\mathrm{RC}}$ & \\
\hline \multicolumn{7}{|l|}{ Family History of IBS } \\
\hline Yes & $62(48.1)$ & $67(51.9)$ & 16.69 & 0 & 2.32 & $1.54-3.49$ \\
\hline No & $113(28.5)$ & $283(71.5)$ & & & & \\
\hline
\end{tabular}

Table 3. Relationship between life-style, chronic conditions, psychological problems and irritable bowel syndrome among paramedical students at King Abdulaziz University.

\begin{tabular}{|c|c|c|c|c|c|c|}
\hline IBS & IBS & No IBS & \multirow{2}{*}{$\mathbf{X}^{2}$} & \multirow{2}{*}{$\mathbf{P}$} & \multirow{2}{*}{ OR } & \multirow{2}{*}{$95 \%$ CI } \\
\hline Variable & No. $\%$ & No. $\%$ & & & & \\
\hline \multicolumn{7}{|l|}{ Physical exercise } \\
\hline Practice & $91(34.2)$ & $175(65.8)$ & 0.19 & 0.67 & 1.08 & $0.75-1.56$ \\
\hline Not practice & $84(32.4)$ & $175(67.6)$ & & & & \\
\hline \multicolumn{7}{|l|}{ Smoking } \\
\hline Yes & $19(41.3)$ & $27(58.7)$ & 1.44 & 0.23 & 1.46 & $0.79-2.70$ \\
\hline No & $156(32.6)$ & $323(67.4)$ & & & & \\
\hline \multicolumn{7}{|l|}{ BMI } \\
\hline Normal & $149(32.7)$ & $306(67.3)$ & 0.53 & 0.468 & 0.82 & $0.49-1.39$ \\
\hline Overweight \& obese & $26(37.1)$ & $44(62.9)$ & & & & \\
\hline \multicolumn{7}{|l|}{ Chronic diseases } \\
\hline Yes & $36(49.3)$ & $37(50.7)$ & 9.75 & 0.002 & 2.19 & $1.33-3.61$ \\
\hline No & $139(30.8)$ & $313(69.2)$ & & & & \\
\hline \multicolumn{7}{|l|}{ Food hypersensitivity } \\
\hline Yes & $32(45.1)$ & $39(54.9)$ & 5.09 & 0.024 & 1.78 & $1.07-2.97$ \\
\hline No & $143(31.5)$ & $311(68.5)$ & & & & \\
\hline \multicolumn{7}{|l|}{ Traveler's diarrhea } \\
\hline Yes & $35(47.9)$ & $38(52.1)$ & 8.15 & 0.004 & 2.05 & $1.24-3.39$ \\
\hline No & $140(31.0)$ & $312(69.0)$ & & & & \\
\hline \multicolumn{7}{|l|}{ Sleeping quality } \\
\hline Poor sleep & $116(39.7)$ & $176(60.3)$ & 12.09 & 0.001 & 1.94 & $1.33-2.83$ \\
\hline Good sleep & $59(25.3)$ & $174(74.7)$ & & & & \\
\hline \multicolumn{7}{|l|}{ Stress } \\
\hline Yes & $112(42.9)$ & $149(57.1)$ & 22.35 & 0 & 2.45 & $1.68-3.57$ \\
\hline No & $62(23.5)$ & $202(76.5)$ & & & & \\
\hline \multicolumn{7}{|l|}{ Anxiety } \\
\hline Borderline \& Morbid & 91 (38.6) & $145(61.4)$ & 5.27 & 0.02 & 1.53 & $1.06-2.21$ \\
\hline Normal & $84(29.1)$ & $205(70.9)$ & & & & \\
\hline \multicolumn{7}{|l|}{ Depression } \\
\hline Borderline \& morbid & $89(42.2)$ & $122(57.8)$ & 12.43 & 0 & 1.93 & $1.34-2.79$ \\
\hline Normal & $86(27.4)$ & $228(72.6)$ & & & & \\
\hline
\end{tabular}


Table 4. Predictors of irritable bowel syndrome among paramedical students from King Abdulaziz University. aOR: Adjusted Odds Ratio.

\begin{tabular}{|l|c|c|c|c|}
\hline Variable & B & $\mathbf{p}$ & $\mathbf{a O R}$ & $\mathbf{9 5 . 0 \%}$ CI \\
\hline Gender (female) & 0.744 & 0.002 & 2.11 & $1.30-3.41$ \\
\hline Stress & 0.606 & 0.003 & 1.83 & $1.12-3.28$ \\
\hline $\begin{array}{l}\text { Depression (morbid \& } \\
\text { borderline) }\end{array}$ & 0.553 & 0.01 & 1.74 & $1.12-2.63$ \\
\hline Family history of IBS & 0.494 & 0.03 & 1.64 & $1.11-2.63$ \\
\hline Sleep quality (poor) & 0.452 & 0.02 & 1.57 & $1.05-2.35$ \\
\hline Constant & \multicolumn{4}{|c|}{-3.865} \\
\hline
\end{tabular}

\section{Discussion}

Up to the best of our knowledge, and based on extensive literature search, the current study may be the first study done about IBS among a large sample of paramedical students from both gender and all grades in Jeddah, KSA.

Our results illustrated that one-third of the paramedical students had IBS, which agrees with the study of Ibrahim et al. [8] from Jeddah. Correspondingly, the prevalence of IBS among students from nursing and medical colleges from China (32.1\%) [13] and Japan (35.5\%) [14]. On the other hand, a lower prevalence of IBS (21.1\%) was reported among 57 nursing and midwifery students from Iran [15]. This discrepancy may be attributed to the smaller sample size from Iran, or because their sample included nursing and midwifery students who may be have less stress, or due to the variations between countries.

Paramedical students who were previously diagnosed as having IBS represented only $24.0 \%$ of those who met Rome III in the current study. This finding is in line with many other previous studies $[8,16,17]$. The present study revealed that IBS-M and IBS- $\mathrm{C}$ were the commonest sub-types. This finding coincides with other studies from Pakistan [10] Saudi Arabia [16] and Egypt [18].

Females in the present study had higher rates of IBS than males, and gender was the first predictor of the condition. This result agrees with the pre-determined association between gender and IBS $[1,7,8,10,14,18]$. This gender difference may be explained by the effect of "Microgenderome" which is related to the possible role of sex-hormones (especially estrogen) on modulation of the gut microbiota, and due to their effects on the peripheral and central regulatory mechanisms of the brain-gut axis. This gender difference may also be due to the differences in responses of both genders to stress $[1,19]$.

Regarding the educational speciality, the highest IBS prevalence was found among nursing and dentistry students. This may be because they may face more stresses compared to others due to their clinical workload, and their dealing with patients. However, students studied clinical nutrition had the lowest IBS prevalence compared to others. This might be due to the nature of their study; with nutritional education about diet and relation to diseases as IBS.

Older students, and those enrolled in higher educational grades in our study were diagnosed to have a more prevalence of IBS compared to others. This may be due to increased study load and stressors during their higher educational levels. Our result agrees with the study of Jeddah [8].

Furthermore, participants with enough and exceeding family income, in the current study, had a lower prevalence of IBS than others, which agree with numerous other studies $[7,16,18]$. This finding may have attributed to the "hygiene hypothesis" and its role in IBS [7].

Students lived with their family had a lower IBS prevalence compared to others $(p>0.05)$, which is in line with other studies
$[7,8,17]$. Living away from family may affect students' lifestyle, decrease their ability to manage stresses, and so become more prone to IBS [7].

IBS prevalence was higher among smokers compared to others in the current study $(p>0.05)$. This agrees with Jeddah study [8]. This can be explained by direct effect of nicotine on gut or being a marker for unfortunate psychological condition [1].

Okami et al. [14] reported absence of an association between practicing physical exercise and IBS, which coincides with our results. Paramedical students who had excessive weight (higher BMI) in our study reported higher IBS prevalence compared to others $(p>0.05)$, which agrees with results from other studies $[8,16]$.

In the current study, participants with positive family history of IBS were more likely to have IBS, which coincides with other studies $[7,8]$. It goes in line also with familial aggregation of IBS cases. Results of twin studies support involvement of genetic risk factors in the pathogenesis of IBS [20].

The enteric nervous system is very sensitive to emotional stresses. Negative emotions can cause alteration of intestinal motor function and might cause IBS symptoms [15]. Similarly, our participants who faced stresses during the six months preceded the study were about twice more likely to have IBS than others. Medical and paramedical students usually face stressful life situations due to their extended times of intensive physical and mental function under high pressure [1]. Lee et al. [21] reported also that stress was associated with IBS. A strong association was reported between mental strains and IBS among university students from Germany [22].

Our results revealed presence of significant associations between IBS with each of anxiety and depression. Depression is also one of IBS predictors. Naeem et al. [10] reported also higher anxiety and depression scores between medical students with IBS from Karachi, Pakistan. Similarly, results of a systematic review and meta-analysis included 10 case-control studies, 2014, illustrated that IBS patients had significantly higher levels of anxiety and depression compared to controls [23]. These findings also agree with the results of many other studies $[8,10,14]$. All these findings can be explained by "the bio-psychological model of IBS, with disruption of the brain-gut pathways" [23].

Our research found that poor sleepers were about twice times more likely to have IBS compared to others. Poor sleep quality may be considered as a risky stressful influence that can disturb the normal biological rhythm, and hence changing the gut motility $[1,16]$. This finding coincides with the results from two previous studies from Jeddah $[8,16]$.

Our participants who had chronic medical conditions were more prone to IBS, which agrees with the results of a systematic review done for studies done between medical students [1].

IBS was found to be associated with history of traveler's diarrhea in our bivariate analysis. Results from a previous meta-analysis, 2015, illustrated presence of a strong association between traveler's diarrhea and Post-infection IBS (PI-IBS) [24]. This can be explained by disruption of gut microbiota due to the infection [25].

Our study revealed that IBS prevalence was higher between participants who had food hypersensitivity compared to others. Many other studies highlighted the association between food intolerance and symptoms of IBS $[8,26,27]$. It was reported from a previous Turkish case-control research that the skin prick tests positivity was more common, and the mean IgE values were higher among IBS cases compared to the controls [26]. 


\section{Conclusion}

One-third of paramedical students were diagnosed as having IBS (Rome III), which is considered a high IBS prevalence. IBS-M and IBS-C were the commonest sub-types. IBS was associated with gender, educational specialty, income, family history of IBS, presence of chronic conditions, food hypersensitivity traveler's diarrhea, poor sleeping, stress, anxiety and depression. After controlling confounding, the predictors of IBS were gender, stresses, depression, poor sleeping and family history of IBS. Screening and management of psychological problems is important. Conduction of stress management and IBS educational programs are recommended. Conduction of similar studies among other health professionals is needed.

\section{Acknowledgment}

Authors thanks all participants in the research and all mangers who facilitated the study.

\section{References}

1. Ibrahim NK (2016) A systematic review of the prevalence and risk factors of irritable bowel syndrome among medical students. Turk J Gastroenterol 27: 10-16. [Crossref]

2. Enck P, Aziz Q, Barbara G, Farmer AD, Fukudo S, et al. (2016) Irritable bowel syndrome. Nat Rev Dis Primers 2: 16014. [Crossref]

3. Chang JY, Talley NJ (2010) Current and emerging therapies in irritable bowel syndrome: from pathophysiology to treatment. Trends Pharmacol Sci 31: 326-334. [Crossref]

4. Malagelada JR, Malagelada C (2016) Mechanism-Oriented Therapy of Irritable Bowel Syndrome. Adv Ther 33: 877-893. [Crossref]

5. Ballou S, Keefer L (2016) The impact of irritable bowel syndrome on daily functioning: Characterizing and understanding daily consequences of IBS. Neurogastroenterol Motil: 29. [Crossref]

6. Canavan C, West J, Card T (2014) The epidemiology of irritable bowel syndrome. Clin Epidemiol 6: 71-80. [Crossref]

7. Costanian C, Tamim H, Assaad S (2015) Prevalence and factors associated with irritable bowel syndrome among university students in Lebanon: Findings from a crosssectional study. World J Gastroenterol 21: 3628-3635. [Crossref]

8. Ibrahim NK, Battarjee WF, Almehmadi SA (2013) Prevalence and predictors of irritable bowel syndrome among medical students and interns in King Abdulaziz University, Jeddah. Libyan J Med. 8: 21287. [Crossref]

9. Wang WE (2012) Clinical Epidemiology-basic principles and practical applications. Beijing: Higher Education Press Publication, pp: 101.

10. Naeem SS, Siddiqui EU, Kazi AN, Memon AA, Khan ST, et al. (2012) Prevalence and factors associated with irritable bowel syndrome among medical students of Karachi, Pakistan: a cross-sectional study. BMC Res Notes 5: 255. [Crossref]

11. Ibrahim N, Al- Kharboush D, El-Khatib L, Al -Habib A, Asali D (2013) Prevalence and Predictors of Anxiety and Depression among Female Medical Students in King Abdulaziz University, Jeddah, Saudi Arabia. Iran J Public Health 42: 726-736. [Crossref]
12. Spira AP, Beaudreau SA, Stone KL, Kezirian EJ, Lui L-Y, et al. (2012) Reliability and Validity of the Pittsburgh Sleep Quality Index and the Epworth Sleepiness Scale in Older Men. J Gerontol A Biol Sci Med Sci 67: 433-439. [Crossref]

13. Okami Y, Nin G, Harada K, Wada S, Tsuji T, et al. (2013) Irritable bowel syndrome in Chinese nursing and medical school students-Related lifestyle and psychological factors. Open J Gastroenterol 3: 55.

14. Okami Y, Kato T, Nin G, Harada K, Aoi W, et al. (2011) Lifestyle and psychological factors related to irritable bowel syndrome in nursing and medical school students. $J$ Gastroenterol 46: 1403-1410. [Crossref]

15. Asadi M, Cheshmeh MGD, Mahmoodi M, Zarea K, Ghomeishi A, et al. (2015) Relationship Between Student QoL With Irritable Bowel Syndrome and Related Factors at Ahvaz Jundishapur University of Medical Sciences Jundishapur Journal of Chronic Disease Care 4: e26624.

16. Ibrahim NK, Al-Bloushy RI, Sait SH, Al-Azhary HW, Al Bar NH, et al. (2016) Irritable bowel syndrome among nurses working in King Abdulaziz University Hospital, Jeddah, Saudi Arabia. Libyan J Med 11: 30866. [Crossref]

17. Mansour-Ghanaei F, Fallah MS, Heidarzadeh A, Jafarshad R, Joukar F, et al. (2011) Prevalence and characteristics of irritable bowel syndrome (IBS) amongst medical students of Gilan Northern Province of Iran. Middle East J Dig Dis 1: 100-105.

18. Darweesh MM, El Hameed MAMA, Hassan YM, El Rheem KAA, Mohamed SA, et al. (2015) The prevalence of irritable bowel syndrome among medical and non-medical Suez Canal University students. Open J Gastroenterol 5: 42.

19. Mulak A, Taché Y, Larauche M (2014) Sex hormones in the modulation of irritable bowel syndrome. World J Gastroenterol 20: 2433-2448. [Crossref]

20. Villani AC, Lemire M, Thabane M, Belisle A, Geneau G, et al. (2010) Genetic risk factors for post-infectious irritable bowel syndrome following a waterborne outbreak of gastroenteritis. Gastroenterology 138: 1502-1513. [Crossref]

21. Lee SP, Sung IK, Kim JH, Lee SY, Park HS, et al. (2015) The effect of emotional stress and depression on the prevalence of digestive diseases. J Neurogastroenterol Motil 21: 273-282. [Crossref]

22. Gulewitsch MD, Enck P, Schwille-Kiuntke J, Weimer K, Schlarb AA (2013) Mental Strain and Chronic Stress among University Students with Symptoms of Irritable Bowel Syndrome. Gastroenterol Res Pract: 206574. [Crossref]

23. Fond G, Loundou A, Hamdani N, Boukouaci W, Dargel A, et al. (2014) Anxiety and depression comorbidities in irritable bowel syndrome (IBS): a systematic review and meta-analysis. Eur Arch Psychiatry Clin Neurosci 264: 651-660. [Crossref]

24. Schwille-Kiuntke J, Mazurak N, Enck P (2015) Systematic review with meta-analysis: post-infectious irritable bowel syndrome after travellers' diarrhoea. Aliment Pharmacol Ther 41: 1029-1037. [Crossref]

25. Collins SM (2016) The Intestinal Microbiota in the Irritable Bowel Syndrome. Int Rev Neurobiol 131: 247-261. [Crossref]

26. Carroccio A, Brusca I, Mansueto P, Soresi M, D'Alcamo A, et al. (2011) Fecal assays detect hypersensitivity to cow's milk protein and gluten in adults with irritable bowel syndrome. Clin Gastroenterol Hepatol 9: 965-971.e3. [Crossref]

27. Uz E, Turkay C, Aytac S, Bavbek N (2007) Risk factors for irritable bowel syndrome in Turkish population: role of food allergy. J Clin Gastroenterol 41: 380-383. [Crossref]

Copyright: (C2018 Ibrahim NK. This is an open-access article distributed under the terms of the Creative Commons Attribution License, which permits unrestricted use, distribution, and reproduction in any medium, provided the original author and source are credited. 